Open Access

\title{
Erratum to: Single centre experience of the application of self navigated 3D whole heart cardiovascular magnetic resonance for the assessment of cardiac anatomy in congenital heart disease
}

Pierre Monney ${ }^{1 \dagger}$, Davide Piccini ${ }^{2,3,4 \dagger}$, Tobias Rutz ${ }^{1}$, Gabriella Vincenti ${ }^{1}$, Simone Coppo ${ }^{3,4}$, Simon C. Koestner ${ }^{1}$, Nicole Sekarski ${ }^{5}$, Stefano Di Bernardo ${ }^{5}$, Judith Bouchardy ${ }^{1}$, Matthias Stuber ${ }^{3,4}$ and Juerg Schwitter ${ }^{1 *}$

Following publication of the original version of this article [1], it was found that Table seven was missing due to a Production error. The table is now provided below as Table 1.

\footnotetext{
* Correspondence: jurg.schwitter@chuv.ch

${ }^{\dagger}$ Equal contributors

'Division of Cardiology and Cardiac MR Center, University Hospital of

Lausanne (CHUV), Lausanne, Switzerland

Full list of author information is available at the end of the article
} 
Table 1 Detection of the origin and proximal course of the coronary arteries

\begin{tabular}{lcccccccc}
\hline Coronary artery & & Total cohort & Quality grade 1 & Quality grade 2 & Quality grade 3 & Quality grade 4 & Quality grade 5 & $P$ \\
& $N=111$ & $N=1$ & $N=10$ & $N=22$ & $N=41$ & $N=37$ \\
\hline LAD & $N(\%)$ & $103(93 \%)$ & $0(0 \%)$ & $6(60 \%)$ & $20(91 \%)$ & $40(98 \%)$ & $37(100 \%)$ & $<0.001$ \\
LCX & $N(\%)$ & $97(87 \%)$ & $0(0 \%)$ & $6(60 \%)$ & $18(82 \%)$ & $37(90 \%)$ & $36(97 \%)$ & $<0.01$ \\
RCA & $N(\%)$ & $109(98 \%)$ & $1(100 \%)$ & $10(100 \%)$ & $21(95 \%)$ & $40(98 \%)$ & $37(100 \%)$ & 0.75 \\
\hline
\end{tabular}

\section{Competing interests}

Davide Piccini, PhD, is an employee of Siemens Healthcare. No financial support from industry was obtained for this study.

\section{Authors' contributions}

PM contributed to the conception and design of the study and to the acquisition of data. He carried out the image quality assessment, the vessel diameter measurement and the segmental anatomical description, and performed the statistical analysis. He drafted the manuscript and participated to its critical revision. DP contributed to the conception and design of the study and to the acquisition of data. He performed the image quality assessment and critically revised the manuscript. TR contributed to the conception and design of the study, acquisition and analysis of the data. He carried out the vessel diameter measurement as second observer and the segmental anatomical description, and critically revised the manuscript. GV contributed to the conception and design of the study and to the acquisition of data. She critically revised the manuscript. SC extracted the parameters of heart rate variability from the raw image data and he critically revised the manuscript. SCK contributed to the conception and design of the study and to the acquisition of data. He critically revised the manuscript. NS contributed to the conception and design of the study. She critically revised the manuscript. SDB contributed to the conception and design of the study. He critically revised the manuscript. JB contributed to the conception and design of the study. She critically revised the manuscript. MS contributed to the conception and design of the study, and to the interpretation of the data. He critically revised the manuscript. JS contributed to the conception and design of the study. He contributed to draft the manuscript and revised it critically. All authors read and approved the final manuscript.

\section{Author details}

'Division of Cardiology and Cardiac MR Center, University Hospital of Lausanne (CHUV), Lausanne, Switzerland. ${ }^{2}$ Advanced Clinical Imaging Technology, Siemens Healthcare, Lausanne, Switzerland. ${ }^{3}$ Department of Radiology, University Hospital and University of Lausanne, Lausanne, Switzerland. ${ }^{4}$ Center for Biomedical Imaging and Center for Cardiovascular Magnetic Resonance Research, University of Lausanne, Lausanne, Switzerland. ${ }^{5}$ Pediatric Cardiology Unit, University Hospital of Lausanne (CHUV), Lausanne, Switzerland

Received: 27 July 2015 Accepted: 27 July 2015

Published online: 06 October 2015

\section{Reference}

1. Monney P, Piccini D, Rutz T, Vincenti G, Coppo S, Koestner S, et al. Single centre experience of the application of self navigated $3 \mathrm{D}$ whole heart cardiovascular magnetic resonance for the assessment of cardiac anatomy in congenital heart disease. J Cardiovasc Magn Reson. 2015;17:55. 